\title{
Aprendizaje Basado en Proyectos en Cinética Química y Catálisis coordinada con otras asignaturas del Grado en Ingeniería Química
}

\author{
María-Fernanda López-Pérez, S.C. Cardona Navarrete, J. Lora García, Vicent \\ Fombuena Borrás \\ Departamento de Ingeniería Química y Nuclear. Universitat Politècnica de València (UPV). Plaça \\ Ferràndiz i Carbonell, s/n 03801 Alcoy, Alicante (Spain). scardona@iqn.upv.es, malope1@iqn.upv.es, \\ jlora@iqn.upv.es, vifombor@upvnet.upv.es
}

\begin{abstract}
As we all know, the aging of teaching staff in universities is a fact, so the teaching methodology we have received and that our students need is very different. However, the passion for this teaching work and the support from Universities with specific program,s are allowing teachers to be trying to implement methodologies to improve motivation and knowledge of our students. One of these methodologies is Project Based Learning (PBL) and not only in a specific subject, but also in several subjects of the University Degrees. In this sense, the Degree in Chemical Engineering of the Universitat Politèctnica de València, Campus d'Alcoi, is trying to coordinate some of its subjects, using this methodology, coordinating a design project of a process related to the Chemical engineering. In this paper, the work will be presented in a specific subject, Chemical Kinetics, 2nd year, and how it has been coordinated with other subjects, for the resolution through PBL of an Engineering problem.
\end{abstract}

Keywords: PBL, Coordination in Chemical Engineering Degree, Activated Carbon Adsorption

\footnotetext{
Resumen

Como todos ya sabemos, el envejecimiento del personal docente en las Universidades es un hecho, con lo que la metodología docente que hemos recibido y la que nuestros alumnos necesitan es muy diferente. Sin embargo, la el pasión a la profesión de docente y los apoyos por parte de las Universidades con programas especificos están permitiendo que los docentes estemos intentando poner en marcha metodologías que encajen con nuestros estudiantes. Una de estas metodologías es el Aprendizaje Basado En Proyectos $(A B P)$ y no solo en una asignatura concreta, sino en varias asignaturas de los Grados Universitarios. En este sentido, el Grado en Ingeniería Química de la Universitat Politèctnica de València, Campus d'Alcoi, está intentando coordinar algunas de sus asignaturas, utilizando esta metodología, coordinando el proyecto en la resolución de un problema de diseño de un proceso relacionado con la Ingeniería Química. En este trabajo se presentará el trabajo en una asignatura concreta, Cinética Química, $2^{\circ}$ curso,
} 
cuatrimestre B, y como se ha coordinado con otras asignaturas, para la resolución mediante un APB de un problema de Ingeniería concreto.

Palabras clave: Aprendizaje basado en proyectos, coordinación, Grado en Ingeniería Química, Proyecto absorción Carbon Activado.

\section{Introducción}

Actualmente nuestros alumnos, pertenecientes a la generación $\mathrm{Z}$, necesitan resultados inmediatos, es decir, obtener un resultado al cuál le vean una utilidad aplicado al mundo que les rodea, no pueden esperar a obtener unos resultados para poder aplicarlos en otras asignaturas, o ver solo la resolución parcial de un problema concreto (Vilanova, N., 2017). Este hecho ha planteado dificultades a los docentes que pertenecemos a una generación en la que se los conceptos que se impartían en las asignaturas de las titulaciones estaban separados siendo en la mayoría de las veces inconexos. Por ello, actualmente, mantener las asignaturas sin ningún tipo de coordinación y objetivo relacionado con algún tema que el alumno pueda experimentar en el mundo real, provoca un rechazo en nuestros estudiantes. En la mayoría de ocasiones la respuesta de los alumnos en las encuestas es que no le han visto la utilidad a los que han estudiado, lo que nos indica que o bien el título no está bien coordinado o los problemas que se resuelven aun siendo complicados no presentan una situación real (LópezGuerrero, M., 2016).

Se caracterizan por ser consumidores multitarea. Les gusta crear contenidos y usan principalmente los medios de mensajería instantánea, debido a su necesidad de inmediatez. No están acostumbrados a esperar, por lo tanto, no les gusta la demostración de los conceptos, sino quieren obtener la utilidad del mismo. Enfocan el trabajo, el aprendizaje y los juegos de forma muy diferente a las generaciones anteriores, tienen información multimedia de imágenes y videos, y la gestionan igual o mejor que si fuera texto; consumen datos de múltiples fuentes; y esperan respuestas instantáneas.

Para motivar a esta generación, debes proporcionarles retos que vayan consiguiendo y que esto tenga una utilidad para ellos: una nota o una utilidad evidente para su futuro profesional. Por ello, en el curso 2017-2018, en el Grado de Ingeniería Química, en la Universitat Politècnica de València, Campus d'Alcoi, se propuso un Proyecto de Innovación y Mejora Educativa (PIME), que pretendía el diseño de problemas reales y abiertos, que engloben diferentes conceptos y que puedan ser utilizados en varias asignaturas del grado y que pueda ser beneficioso tanto para la motivación del alumno, como para la coordinación del profesorado. El proyecto elegido fue "Diseño de una columna de carbón activado para el tratamiento de colorantes textiles", siendo el curso 18-19, el elegido para empezar a plantear los diferentes proyectos a realizar en cada una de las asignaturas (López-Perez, MaríaFernanda, 2014,2016,2018).

Durante este curso, cada profesor ha presentado una ficha con las actividades que iba a realizar, los conceptos que necesitaba de las que le precedían y que asignaturas necesitarían sus conceptos. Aunque el objetivo final, es coordinar todas las asignaturas que han 
participado en el PIME, alrededor del mismo proyecto y que el alumno lo trabaje, durante este curso, se han puesto en marcha de forma individual, sin que el alumno pueda relacionar las actividades con un proyecto global, ya que, se necesita un curso o varios para optimizar tanto las actividades como la evaluación de las mismas.

El siguiente paso, una vez, cada asignatura tenga claras las actividades será implementar el proyecto en el Grado con coordinación entre las asignaturas, para que el alumno lo trabajo en los 4 cursos.

\section{Objetivos}

En este trabajo se presenta una parte del proyecto docente de la asignatura Cinética Química y Catálisis en el Grado de Ingeniería Química en la UPV Campus d'Alcoi. El objetivo es presentar la metodología de Aprendizaje Basado en Proyectos en una actividad de dicha asignatura, para la resolución de la parte correspondiente al proyecto "Diseño de una Torre de Adsorción con Carbón activado para la eliminación de Colorantes Textiles" y la relación que se deberá establecer con las asignaturas que participan en dicho proyecto.

Con esta actividad se pretende aumentar la motivación de los alumnos y que sean capaces de enfrentarse a un problema real que pueden encontrar en su futuro profesional.

\section{Desarrollo de la Innovación}

Para desarrollar cualquier innovación educativa, el primer paso es establecer los objetivos que pretendemos alcanzar, en el caso que nos ocupa, el principal objetivo es que el alumno alcance los conocimientos y las competencias que nos marca el documento Verifica, con una metodología que sea capaz de motivarlo y con la que pueda utilizar los conocimientos adquiridos en otras asignaturas.

La actividad que presentamos está enmarcada dentro de la asignatura Cinética Química y Catálisis, perteneciente al segundo curso, y cuatrimestre B. Durante la primera parte del curso, los alumnos han adquirido los conceptos más básicos de la asignatura, como puede ser la obtención de una ecuación cinética a partir de los datos experimentales. En la segunda parte de la asignatura, y teniendo en cuenta que la catálisis química es importante en la industria (el $60 \%$ de los productos químicos se sintetizan por procesos catalíticos, el $70 \%$ de los procesos químicos de fabricación son catalíticos, más del $99 \%$ de la producción mundial de gasolina ocurre a través del craqueo catalítico de fracciones del petróleo y de otras reacciones catalíticas y más del $90 \%$ de los procesos industriales nuevos son catalíticos), nos pareció adecuado que una parte importante del cuatrimestre, estuviera dedicado a este tipo de cinéticas.

El problema de esta asignatura, es que no podíamos estar directamente relacionados con el proyecto de diseño de una torre de adsorción, ya que, en ésta, no hay reacción química. Sin embargo, en las reacciones catalíticas es necesaria el proceso de adsorción (Fig. 1), por lo que podríamos relacionar dicha columna con los conceptos que se estudian en cinética. De 
esta forma, el alumno, aunque en los cursos posteriores no diseñe un reactor químico de catálisis, y no utilice directamente los conceptos que se imparten en esta asignatura, es capaz de ver y relacionar procesos que ocurren en varios procesos industriales.

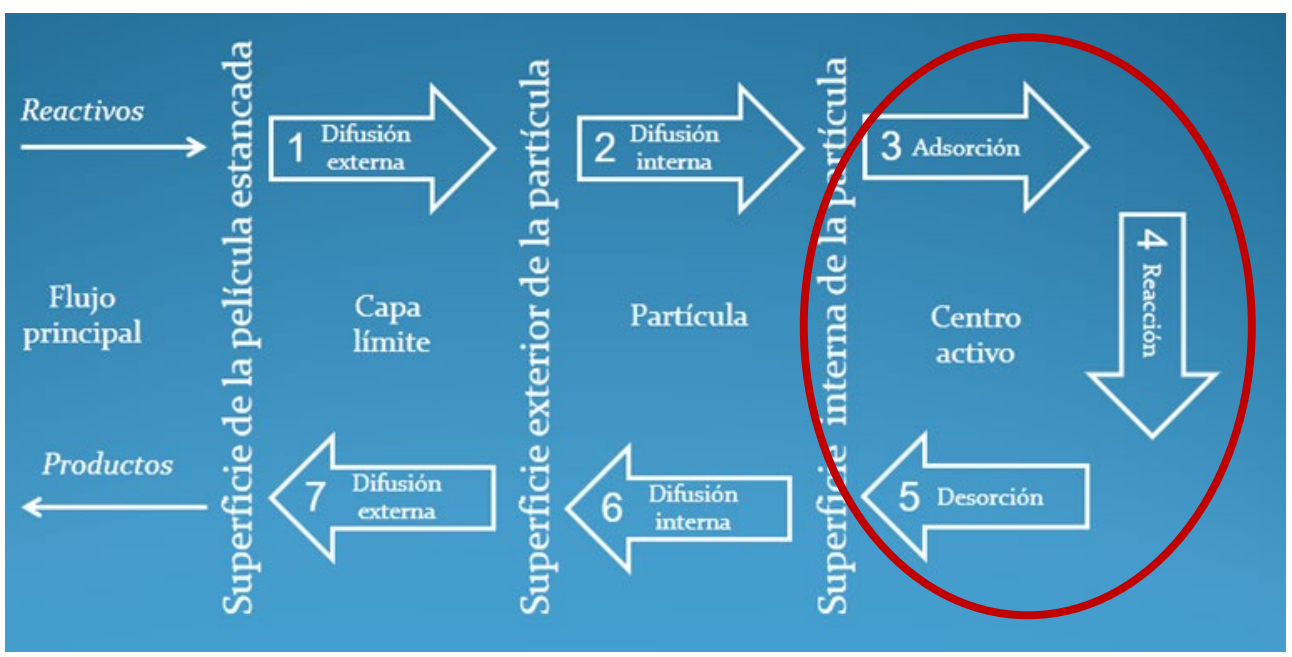

Fig. 1 Procesos en una reacción catalítica

Los conceptos en los que la asignatura se centrarían, serían los rodeados en la figura por el círculo, por lo que es importante para el proyecto, que estos se hayan adquirido anteriormente y que, en esta parte de la asignatura, se pudieran combinar para obtener la ecuación cinética de una reacción de catálisis.

La reacción química se ha estudiado en la primera parte de la asignatura, mientras que el proceso de adsorción puede impartirse en la asignatura de Experimentación en Ingeniería I. Además, en el proceso de catálisis química, existe una transferencia de materia, por lo que, también se podría relacionar con la asignatura de Transferencia de Materia.

Como vemos, esta actividad debe relacionarse con otras asignaturas del Grado, aunque en su coordinación se debe tener en cuenta que, en la UPV, las tres, se imparten en el segundo curso y en el mismo cuatrimestre. Una distribución en el tiempo adecuada obliga a que la parte de la difusión que se imparte en transferencia y los conceptos de isoterma de adsorción hayan finalizado antes del comienzo de la parte de reacción catalítica. Teniendo en cuenta que el cuatrimestre en la UPV tiene una duración de 15 semanas, y que la cinética química empieza en la semana 8 , sería recomendable que los docentes se hayan podido coordinar para que los conceptos tengan la secuenciación adecuada.

Una vez planteados los objetivos y las asignaturas relacionadas los pasos para poder llevar a cabo el proyecto en la asignatura de Cinética Química y Catálisis son:

1. Presentación de un problema real en la industria.

2. Preparación de grupos de trabajo.

3. Plantear las actividades a resolver con la metodología Proyecto basado en problemas. 
4. Plantear sistema de evaluación.

5. Analizar resultados.

\section{Presentación del problema}

El primer paso es presentar a los alumnos el proyecto. Será un proyecto único para todos los alumnos, ya que se realizará en las horas de clase. Esto nos permite que el mismo problema pueda utilizarse durante varios cursos, evitando copias de un año a otro.

El proyecto es: Obtención de una ecuación cinética para la oxidación de COV’s (Etanol y tolueno) utilizando óxidos de cerio soportados en carbón activado.

Lo importante es que el alumno relacione este problema con su vida real, y en este caso, es la eliminación de los COV's que presentan un problema medioambiental debido a que estos compuestos están presentes en las emisiones de vehículos procedentes de la combustión incompleta y las emisiones industriales principalmente de disolventes orgánicos procedentes de numerosos procesos industriales, tales como la industria gráfica (tolueno, n-hexano, propanol), la industria metalúrgica (cetonas, xilenos), la industria química y farmacéutica, la industria electrónica (alcanos, acetatos), la industria alimentaria (etanol de las fermentaciones y de aminas del procesado de alimentos).

Durante la primera clase se plantea el problema y se le muestra un video donde expliquen qué son los COV's y problemas medioambientales que generan.

\section{Preparación de grupos de trabajo}

Los grupos o equipos de trabajo serán de cuatro integrantes, para evitar que si algún compañero abandona la asignatura no deje el grupo demasiado pequeño para el volumen de trabajo. Los grupos serán formados por ellos, solo en los casos en los que haya algún conflicto el profesor intervendrá en los equipos.

Se pedirá que haya un portavoz diferente en cada una de las sesiones (2 horas cada sesión) para que al final de la clase, sea esta persona la que ofrezca al profesor los resultados que se han obtenido.

Se observará los roles que tiene cada alumno y como el trabajo se realizará en el aula se observará que todos los integrantes trabajan.

\section{Actividades a resolver}

El proyecto tendrá las partes de un proyecto de investigación ya que en esta asignatura no podemos plantear un proyecto de ingeniería. Así, las partes que tendremos serían:

1. Antecedentes o introducción. Deben buscar el estado del arte.

2. Objetivos. El objetivo será obtener la ecuación cinética de la oxidación de los COV's 
3. Experimental. Se les proporcionará información para encontrar de artículos de investigación con los equipos que se necesitan para la realización de los experimentos. Buscarán videos de los experimentales.

4. Resultados. Con datos proporcionados por el docente se realizarán los cálculos.

5. Conclusiones. Se presentarán los resultados y las conclusiones.

Todas las actividades serán entregadas en la plataforma PoliformaT en el apartado de Tareas que se cerrará al acabar la clase.

En la siguiente tabla se muestra la temporalización de la asignatura (Tabla 1)

Tabla 1. Temporalización de las actividades a realizar en el proyecto

\begin{tabular}{|c|c|c|}
\hline $\begin{array}{l}N^{0} \text { de } \\
\text { Sesión }\end{array}$ & Actividad Presencial & Actividad no presencial \\
\hline 1 & $\begin{array}{l}\text { Presentación proyecto. } \\
\text { Planteamiento de bases y } \\
\text { metodología a seguir. } \\
\text { Búsqueda de problemas } \\
\text { ambientales producidos por los } \\
\text { COV's y metodología para la } \\
\text { eliminación de los mismos. } \\
\text { Búsqueda de bibliografía } \\
\text { mediante bases de datos. }\end{array}$ & $\begin{array}{l}\text { Realización de los antecedentes en el } \\
\text { estado del arte. Parte de los problemas y } \\
\text { objetivos. }\end{array}$ \\
\hline 2 & $\begin{array}{l}\text { Presentación por parte del } \\
\text { profesor de las reacciones de } \\
\text { catálisis. } \\
\text { Búsqueda bibliográfica de } \\
\text { reacciones de catálisis } \\
\text { relacionadas con los COV's }\end{array}$ & $\begin{array}{l}\text { Realización del estado del arte. Parte de } \\
\text { los catalizadores utilizados y objetivos } \\
\text { del proyecto. }\end{array}$ \\
\hline 3 & $\begin{array}{l}\text { Búsqueda de los diferentes } \\
\text { equipos y parámetros } \\
\text { importantes a tener en cuenta en } \\
\text { las reacciones catalíticas. }\end{array}$ & $\begin{array}{c}\text { Realización del resumen de los diferentes } \\
\text { parámetros a medir y los equipos } \\
\text { utilizados. }\end{array}$ \\
\hline 4 & $\begin{array}{l}\text { Se les proporcionarán datos de } \\
\text { las isotermas de adsorción con } \\
\mathrm{N} 2 \text { para obtener los parámetros } \\
\text { texturales. Cálculo isoterma }\end{array}$ & $\begin{array}{l}\text { Justificación de obtención de la isoterma. } \\
\text { Justificación de la importancia de los } \\
\text { parámetros texturales. }\end{array}$ \\
\hline 5 & $\begin{array}{l}\text { Se les proporcionará datos de } \\
\text { conversiones a diferentes } \\
\text { temperaturas para la oxidación }\end{array}$ & Presentación de cálculos en informe \\
\hline
\end{tabular}




\begin{tabular}{|c|c|c|}
\hline & $\begin{array}{l}\text { de Etanol como modelo de } \\
\text { COV's. Calculo de cinética }\end{array}$ & \\
\hline 6 & $\begin{array}{l}\text { Se les proporcionará datos de } \\
\text { conversiones a diferentes } \\
\text { temperaturas para la oxidación } \\
\text { del Tolueno como modelo de } \\
\text { COV's. Calculo de cinética }\end{array}$ & Presentación de cálculos en informe \\
\hline 7 & Preparar las conclusiones & $\begin{array}{l}\text { Realizar el informe y una presentación } \\
\text { en Power Point para la evaluación oral. }\end{array}$ \\
\hline 8 & Evaluación: Presentación oral & \\
\hline 9 & $\begin{array}{l}\text { Evaluación: Resolución de un } \\
\text { problema relacionado con el } \\
\text { trabajo realizado en el proyecto. }\end{array}$ & \\
\hline
\end{tabular}

\section{Evaluación}

El proyecto constará de dos evaluaciones, una presentación oral, donde se elegirá al azar el portavoz que realizará dicha presentación. Esta metodología permite que todos los integrantes del grupo se hayan preparado las presentaciones, pero no sepan a priori quien va a realizar la exposición.

La segunda evaluación constará de un problema donde se pedirá la obtención de una ecuación cinética de una reacción de catálisis similar a la realizada durante el proyecto, pero naturalmente, más sencillo y que se pueda resolver en dos horas. Esto nos asegura que los alumnos trabajen, no solo en grupo, sino también de forma individual.

La evaluación quedará puntuada de la siguiente forma, el trabajo en grupo (30\%) y la resolución del problema de forma individua (30\%), tal y como se indica en la tabla 2.

Tabla 2. Ponderación de la evaluación

\begin{tabular}{|c|c|}
\hline & Puntuación evaluación \\
\hline $\begin{array}{c}\text { Parte trabajo 1: Introducción, búsqueda de } \\
\text { información teórica y conclusiones }(1,2,3,7)\end{array}$ & $6 \%$ \\
\hline $\begin{array}{c}\text { Parte trabajo 2: Resolución Isoterma } \\
\text { adsorción N2 (4) }\end{array}$ & $\mathbf{9 \%}$ \\
\hline $\begin{array}{c}\text { Parte trabajo 2: Resolución reacción } \\
\text { oxidación COV's (5,6) }\end{array}$ & $\mathbf{6 \%}$ \\
\hline Parte trabajo 3: Presentación oral (8) & $\mathbf{3 0 \%}$ \\
\hline Problema a resolver individual (9) & \\
\hline
\end{tabular}




\section{Resultados}

En cuanto a los resultados obtenidos hasta ahora (recordemos que hemos puesto en marcha la experiencia en el curso 18-19 semestre B, que acaba en junio), cabe destacar, que esta metodología de enseñanza me ha permitido como docente, interactuar de forma más activa con el aprendizaje de los alumnos y detectar que dificultades tenían en la resolución de los problemas. Además, y debido a que la Tarea (lugar donde debían de entregar los trabajos) se cerraba al terminar la sesión obligaba a los alumnos a estar en tensión y trabajar, ya que al final el $\%$ en la nota es significativo.

Esta metodología nos ha permitido trabajar diversos contenidos favoreciendo el aprendizaje, aunque en un principio, pensabamos que sería motivadora para los alumnos, ya que son el papel protagonista de todo el proceso, la experiencia durante este curso, nos ha confirmado que los alumnos no están aconstumbrados a este tipo de trabajos, siendo para ellos un esfuerzo en algunas ocasiones demasiado grande, y al final no se si ha sidouna experiencia para ellos motivadora. El curso siguiente se puede intentar captar más su atención, llevándolos a un laboratorio o una industria en la que se utilicen catalizadores de verdad para que puedan ver la utilidad real de lo que se explica en clase.

En cuanto a los grupos, decir que, aunque 4 alumnos son en algunas ocasiones demasiados (1-2 trabajan y 2-3 miran),el número ha sido acertado, ya que hay algunos grupos en los que algunos integrantes han abandonado la asignatura y no obliga a recolocar al que se queda solo en otro grupo o que trabaje individualmente.

Con la evaluación de las tareas se ha observado que hasta la fecha actual del curso los resultados en cuanto a conocimientos han aumentado, ya que en las sesiones en las que no se realiza el trabajo la resolución de los problemas ha sido más satisfactoria.

Falta realizar las encuestas al alumnado (antes del examen) y las encuestas al profesorado respecto a esta metodología y los resultados del problema individual.

\section{Conclusiones}

La metodología de aprendizaje basado en proyectos nos permite que los alumnos desarrollen los conocimientos adquiridos en otras asignaturas y que puedan relacionar los conceptos con procesos industriales o problemas reales. Se enfrentan a los problemas de forma diferente, ya que no tienen clases magistrales.

Todavía falta por evaluar los resultados en las evaluaciones de los alumnos para comparar con los años anteriores. Durante el mes de junio se estudiarán los resultados.

Le proyecto presentado en esta asignatura ayuda a coordinar varias asignaturas del Grado en Ingeniería Química 


\section{Referencias}

LÓPEZ-GUERRERO, M., BLANCO, A., SERRANO, J., (2017). "Valoración de la utilidad de la química por estudiantes de Ingeniería Mecánica : Efecto de una propuesta didáctica" en Educación Química, 28, 14-21.

LOPEZ-PEREZ, MARIA-FERNANDA (2014) PIME "Utilización de MATLAB como estrategia didáctica y de coordinación horizontal y vertical entre asignaturas del Grado de Ingeniería Química. ”. Proyectos Innovación y Mejora Educativa Universitat Politècnica de València.

LOPEZ-PEREZ, MARIA-FERNANDA, CARDONA, S.C., LORA, J., ABAD A. (2016). MATLAB as a tool as Analysis and Problem Solving Competency Development in Chemical Engineering Degree using MATLAB. Multidisciplinary Journal for Education, Social and Technological Sciences EISSN: 2341-2593 http://dx.doi.org/10.4995/muse.2016.4623.

LOPEZ-PEREZ, MARIA-FERNANDA, CARDONA, S.C., LORA, J., ABAD, A., TORREGROSA, J.I. (2015). Resultados del Proyecto de Innovación y Mejora Educativa. Utilización de MATLAB como estrategia didáctica y de coordinación horizontal y vertical entre asignaturas del Grado de Ingeniería Química.. Comunicación en Congreso IN-Red. 2015. http://inred.blogs.upv.es/

VILANOVA, N, ORTEGA, I. (2017). Generación Z. Todo lo que necesitas saber sobre los jóvenes que han dejado viejos a los millennials. Spain Plataforma 2017. ISBN : 9788417114329 